\title{
Domain Wall Motions in Ferromagnetic Thin Film Induced by Laser Heating Pulse
}

\author{
Hyun Soon Park*
}

Department of Materials Science and Engineering, Inha University, Incheon 22212, Korea

*Correspondence to:

Park HS,

(iD) https://orcid.org/0000-0002-1039-7334 Tel: +82-32-860-7533

Fax: +82-32-862-5546

E-mail: hsparkinha@inha.ac.kr

Received December 21, 2018

Revised December 26, 2018

Accepted December 26, 2018
Soft ferromagnetic materials are utilized for various electromagnetic devices such as magnetic recording heads and magnetic shielding. In situ observation of magnetic microstructures and domain wall motions are prerequisite for understanding and improving their magnetic properties. In this work, by the Fresnel (out-of-focus) method of Lorentz microscopy, we observe the domain wall motions of polycrystalline Ni/Ti thin film layers triggered by single-shot laser pulse. Random motions of domain walls were visualized at every single pulse.

Key Words: Lorentz microscopy, Ferromagnetic thin film, Domain wall, Laser heating pulse, TEM
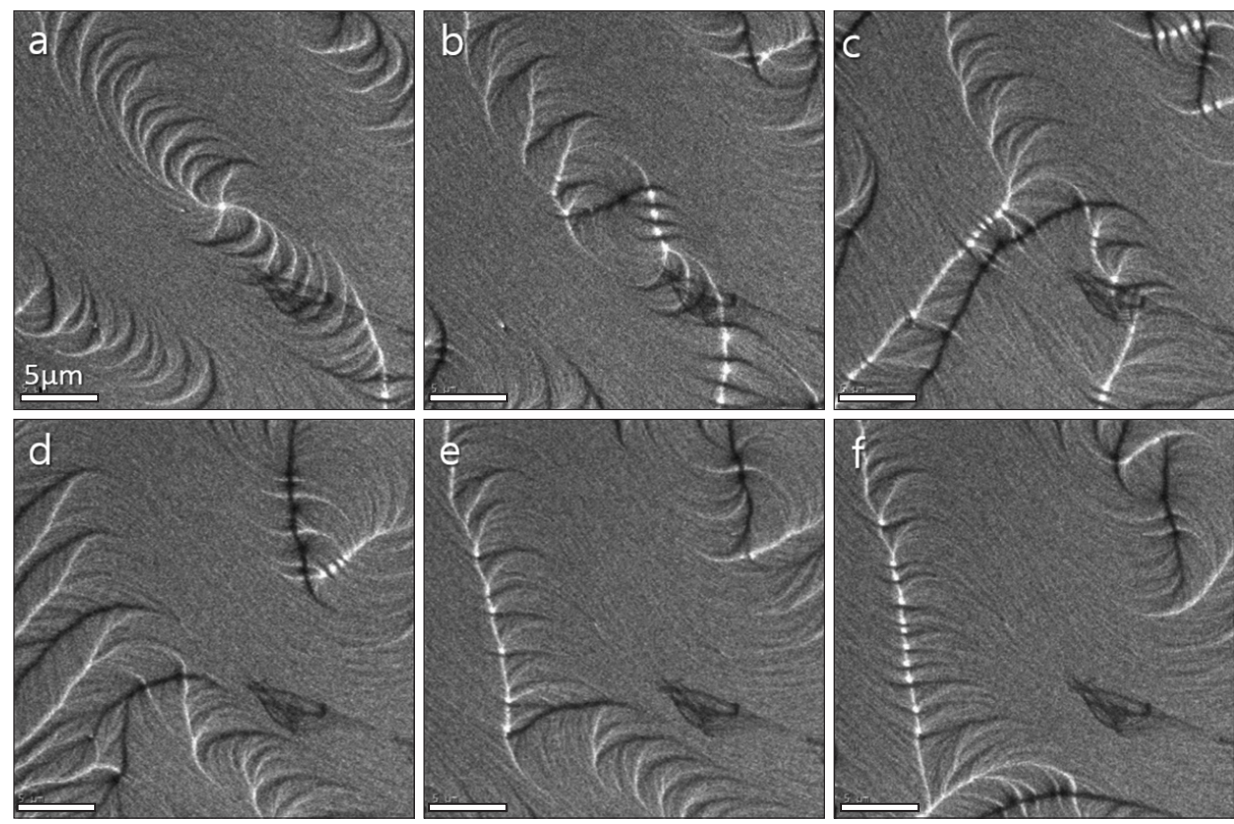

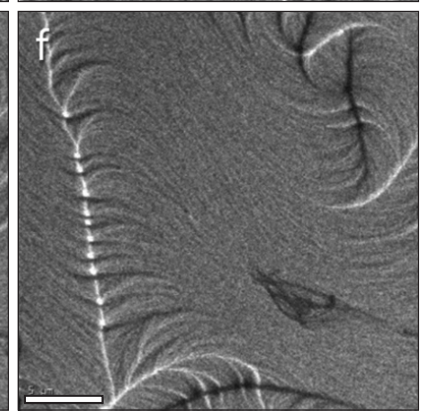

Domain wall motions (Chikazumi, 1997) were in situ observed by Lorentz microscopy (Caltech's second-generation ultrafast electron microscope: Zewail et al., 2010). Polycrystalline thin film layers of $\mathrm{Ni}$ on $\mathrm{Ti}(30 \mathrm{~nm} / 30 \mathrm{~nm})$ were deposited sequentially by electron beam evaporation onto a $\mathrm{Si}_{3} \mathrm{~N}_{4}$ membrane ( $15 \mathrm{~nm}$ thick, $0.25 \times 0.25 \mathrm{~mm}^{2}$ window). To trigger the domain wall motions,

(a) This is an open-access article distributed under the terms of the Creative Commons Attribution Non-Commercial License (http://creativecommons.org/licenses/by-nc/4.0) which permits unrestricted noncommercial use, distribution, and reproduction in any medium, provided the original work is properly cited.

Copyrights (c) 2018 by Korean Society of Microscopy 


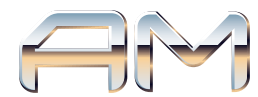

single-shot laser pulse of about $0.24 \mu \mathrm{J}$ (Q-switched Nd:YAG laser at $532 \mathrm{~nm}$ ) was applied to the specimen. The laser pulse was estimated to be $50 \mu \mathrm{m}$ full-width-at-half-maximum Gaussian spot size. Lorentz microscopy images were taken under the overfocused condition $(+4 \mu \mathrm{m})$ in low magnification mode with the main objective lens turned off. In the over-focused condition, domain walls (DWs) appear as white and black lines depending on the sense of rotation of the magnetization. Applying every single heating-pulse (a f), the domain walls (cross-tie walls, vortex, magnetic ripples) were changed severely because of soft magnetic properties, meaning that the domain wall motions are irreversible. To make the domain wall motions reversible, we need to control the sample dimension, laser fluences, magnetic properties (Graef et al., 2001; Lee et al., 2017; Park et al., 2010).

\section{CONFLICT OF INTEREST}

No potential conflict of interest relevant to this article was reported.

\section{ACKNOWLEDGMENTS}

This study was supported by Inha University Research Grant (INHA-55432).

\section{REFERENCES}

Chikazumi S (1997) Physics of ferromagnetism. Oxford Science Publications, Oxford.

Zewail A H, Thomas J M (2010) 4D electron microscopy: imaging in space and time. Imperial College Press, London.

Graef M D, Zhu Y (2001) Magnetic imaging and its applications to materials. Academic Press, London.
Park H S, Baskin J P, Zewail A H (2010) 4D Lorentz electron microscopy imaging: magnetic domain wall nucleation, reversal, and wave velocity. Nano Letters 10, 3796-3803.

Lee S J, Lee H J, Song K, Choi S Y, Park H S (2017) In-situ observation of domain wall motion in electroplated Ni80-Fe20 thin film by Lorentz TEM and DPC imaging. Journal of Magnetics 22, 563-560. 